TRANSACTIONS OF THE

AMERICAN MATHEMATICAL SOCIETY

Volume 355, Number 5 , Pages 2115-2137

S 0002-9947(02)03189-6

Article electronically published on November 14, 2002

\title{
OSCILLATION AND VARIATION FOR SINGULAR INTEGRALS IN HIGHER DIMENSIONS
}

\author{
JAMES T. CAMPBELL, ROGER L. JONES, KARIN REINHOLD, AND MÁTÉ WIERDL
}

\begin{abstract}
In this paper we continue our investigations of square function inequalities in harmonic analysis. Here we investigate oscillation and variation inequalities for singular integral operators in dimensions $d \geq 1$. Our estimates give quantitative information on the speed of convergence of truncations of a singular integral operator, including upcrossing and $\lambda$ jump inequalities.
\end{abstract}

\section{Introduction AND STATEMENT OF RESUlts}

In 4, we obtained estimates for the oscillation and variation of the classical Hilbert transform and related convolution operators acting on $L^{p}(\mathbb{R})$. In particular, if

$$
H_{\epsilon} f(x)=\int_{|t|>\epsilon} \frac{f(x-t)}{t} d t
$$

then we considered the variational operator

$$
V_{\varrho} H f(x)=\sup _{\left(\epsilon_{i}\right) \searrow 0}\left(\sum_{i=1}^{\infty}\left|H_{\epsilon_{i}} f(x)-H_{\epsilon_{i+1}} f(x)\right|^{\varrho}\right)^{\frac{1}{\varrho}},
$$

where the pointwise supremeum is taken over all sequences $\left(\epsilon_{i}\right)$ that are decreasing to zero. Letting $\left(I_{i}\right)$ denote the interval $\left[\epsilon_{i+1}, \epsilon_{i}\right)$, and denoting by $H_{I} f(x)$ the operator

$$
\int_{|t| \in I} \frac{f(x-t)}{t} d t
$$

we see that the operator $V_{\varrho} f(x)$ can be expressed more conveniently as

$$
V_{\varrho} f(x)=\sup _{\left\{I_{i}\right\}}\left(\sum_{i=1}^{\infty}\left|H_{I_{i}} f(x)\right|^{\varrho}\right)^{\frac{1}{\varrho}},
$$

where the pointwise supremum is taken over all sequences of intervals $\left\{I_{i}\right\}$ that partition $(0, \infty)$. We showed that $\left\|V_{\varrho} H f\right\|_{p} \leq c(p, \varrho)\|f\|_{p}$ for $1<p<\infty$, and that

$$
m\left\{x: V_{\varrho} f(x)>\lambda\right\} \leq \frac{c(\varrho)}{\lambda}\|f\|_{1} .
$$

Received by the editors April 4, 2002 and, in revised form, August 19, 2002.

2000 Mathematics Subject Classification. Primary 42B25; Secondary 40A30.

Key words and phrases. Singular integrals, square functions, variation, oscillation, upcrossing inequalities, jump inequalities.

The second author was partially supported by NSF Grant DMS - 9302012 .

The fourth author was partially supported by NSF Grant DMS—9500577.

(C)2002 American Mathematical Society 
In this paper we extend these and related results to singular integral operators acting on $L^{p}\left(\mathbb{R}^{d}\right), d \geq 1$. This work may be viewed as part of a larger program of investigations into square function inequalities in harmonic analysis and ergodic theory; see, for example, ([4], [8], [9], 10]).

The higher-dimensional oscillation and variation operators are defined as follows. For an interval $I \subset(0, \infty)$, let $A_{I}$ denote the annulus $\left\{x \in \mathbb{R}^{d}|| x \mid \in I\right\}$, where $|x|$ is the Euclidean norm of $x$. For a singular real-valued kernel $K$ on $\mathbb{R}^{d}$ (satisfying conditions to be specified later), define the transformation $K_{I}$ acting on locally integrable functions $f: \mathbb{R}^{d} \rightarrow \mathbb{R}$ by

$$
K_{I} f(x)=\int_{A_{I}} K(y) f(x-y) d y .
$$

Definition 1.1. Let $\mathcal{I}=\left\{I_{k}\right\}$ be a fixed countable partition of $(0, \infty)$ into intervals of the form $[a, b), a<b$. Denote by $\mathcal{L}_{k}$ the collection of subintervals of $I_{k}=[a, b)$ of the form $[a, c)$, where $a<c \leq b$. Define the oscillation operator $O=O_{\mathcal{I}}$ by

$$
O(K f)(x)=\left(\sum_{k} \sup _{L \in \mathcal{L}_{k}}\left|K_{L} f(x)\right|^{2}\right)^{\frac{1}{2}},
$$

where the supremum is taken pointwise.

Definition 1.2. Define the full variation operator $V_{\varrho}$, for $\varrho>2$, as

$$
V_{\varrho}(K f)(x)=\left(\sup _{\left\{I_{i}\right\}} \sum_{i}\left|K_{I_{i}} f(x)\right|^{\varrho}\right)^{1 / \varrho},
$$

where the pointwise supremum is taken over all partitions $\left\{I_{i}\right\}$ of $(0, \infty)$ into nondegenerate disjoint intervals of the form $[a, b)$.

Although it may not be immediately clear, these operators are measurable. See the comment just after the statement of Theorem 1.2 in [4].

The main results of this paper are the strong $L^{p}$ estimates $(p>1)$ and weak $L^{1}$ estimates for the operators $f \rightarrow O(K f)$ and $f \rightarrow V_{\varrho}(K f)$ given by the following two theorems and the subsequent corollary. Here, and throughout the paper, $|B|$ denotes the Lebesgue measure of a set $B \subset \mathbb{R}^{d}, \Sigma$ (or $\Sigma_{d-1}$ ) denotes the unit sphere $\{|x|=1\} \subset \mathbb{R}^{d}$, and $d S$ denotes Lebesgue surface measure on $\Sigma$.

Theorem A. Let $K: \mathbb{R}^{d} \backslash\{0\} \rightarrow \mathbb{R}$ have the following properties:

$$
\begin{aligned}
& K \text { is homogeneous of degree }-d, \\
& K \log ^{+} K \text { is integrable on the sphere } \Sigma, \\
& \int_{\Sigma} K(x) d S(x)=0 .
\end{aligned}
$$

Then the operator

$$
f \rightarrow O(K f)
$$

and, for $\varrho>2$, the operators

$$
f \rightarrow V_{\varrho}(K f)
$$

are bounded on $L^{p}\left(\mathbb{R}^{d}\right)$ for $p>1$. That is, for $1<p<\infty$ there exist constants $C_{p}$, depending only upon $p$ and perhaps the dimension $d$, and not the partition $\mathcal{I}$, so that

$$
\|O(K f)\|_{L^{p}\left(\mathbb{R}^{d}\right)} \leq C_{p}\|f\|_{L^{p}\left(\mathbb{R}^{d}\right)}, \quad f \in L^{p}\left(\mathbb{R}^{d}\right),
$$


and for $1<p<\infty$, and $\varrho>2$, there exist constants $C_{\varrho, p}$, depending only upon $\varrho$ and $p$ and the dimension $d$, so that

$$
\left\|V_{\varrho}(K f)\right\|_{L^{p}\left(\mathbb{R}^{d}\right)} \leq C_{\varrho, p}\|f\|_{L^{p}\left(\mathbb{R}^{d}\right)}, \quad f \in L^{p}\left(\mathbb{R}^{d}\right) .
$$

Our next theorem requires the following definition.

Definition 1.3. Let $\mathcal{I}=\left\{I_{1}, I_{2}, \ldots, I_{n}\right\}$ be a finite collection of subintervals of $(0, \infty)$, each of the form $[a, b)$ for some $a<b$. (In particular, they need not be disjoint.) For $\varrho \geq 2$, define a sublinear transformation $\mathcal{W}_{\varrho}$ on locally integrable functions by

$$
\mathcal{W}_{\varrho} f(x)=\left(\sup _{\left\{I_{i_{1}}, I_{i_{2}}, \ldots, I_{i_{j}}\right\}} \sum_{k}\left|K_{I_{i_{k}}(x)} f(x)\right|^{\varrho}\right)^{1 / \varrho},
$$

where the pointwise supremum is taken over all pairwise disjoint subcollections $\left\{I_{i_{1}}, I_{i_{2}}, \ldots, I_{i_{j}}\right\}$ of $\mathcal{I}$.

Theorem B. Let $K: \mathbb{R}^{d} \rightarrow \mathbb{R}$ satisfy

$$
\begin{aligned}
|K(x)| & \leq \frac{A}{|x|^{d}}, & & |x|>0, \\
\int_{|x| \geq 2|y|}|K(x+y)-K(x)| d x & \leq c, & & |y|>0,
\end{aligned}
$$

where $A$ and $c$ are fixed constants. Suppose that for the collection $\mathcal{I}$ as in Definition 1.3. and some $\varrho \geq 2$, the operator $f \rightarrow \mathcal{W}_{\varrho} f$ is type $(2,2)$. That is, suppose there exists a constant $c_{\varrho}$, depending only upon $\varrho$ and the dimension $d$, so that

$$
\left\|\mathcal{W}_{\varrho} f(x)\right\|_{L^{2}\left(\mathbb{R}^{d}\right)} \leq c_{\varrho}\|f\|_{L^{2}\left(\mathbb{R}^{d}\right)}, \quad f \in L^{2}\left(\mathbb{R}^{d}\right) .
$$

Then there exists a constant $C=C_{\varrho}$, which also may depend upon $A$ and $c$, but not on $f$, so that

$$
\left|\left\{x: \mathcal{W}_{\varrho} f(x)>\alpha\right\}\right| \leq \frac{C_{\varrho}}{\alpha}\|f\|_{L^{1}\left(\mathbb{R}^{d}\right)}, \quad f \in L^{1}\left(\mathbb{R}^{d}\right) .
$$

Corollary 1.4. Let $K$ satisfy the conditions (1.2)-(1.4), (1.7) and (1.8). Then we have the estimates

$$
|\{x:|O(K f)(x)|>\alpha\}| \leq \frac{C_{1}}{\alpha}\|f\|_{L^{1}\left(\mathbb{R}^{d}\right)}, \quad f \in L^{1}\left(\mathbb{R}^{d}\right) .
$$

and, for $\varrho>2$,

$$
\left|\left\{x: V_{\varrho}(K f)(x)>\alpha\right\}\right| \leq \frac{C_{\varrho}}{\alpha}\|f\|_{L^{1}\left(\mathbb{R}^{d}\right)}, \quad f \in L^{1}\left(\mathbb{R}^{d}\right) .
$$

Some remarks are in order.

Remark 1.5. Corollary 1.4 follows from Theorems $\mathrm{A}$ and B. The relatively straightforward proof is given at the end of Section 4. An added benefit of the proof is that one sees directly that the operators $\mathcal{V}_{\varrho}$ and $O$ are measurable.

Remark 1.6. There are still some open questions regarding exactly what conditions should be placed on our singular integral operators in order to obtain a weak type $(1,1)$ result for the variation operator. In particular, if we let $\Omega(x)$ denote the function given by $K(x)$ restricted to the unit sphere, then condition 1.7 implies that $\Omega \in L^{\infty}$. This condition is used in the proof of Theorem $\mathrm{B}$, and it is not obvious how to relax it. However, the classical weak type result for these singular 
integrals involves only knowing that $\Omega \in L \log L$ (see [6]). An earlier result involved an entropy condition on $\Omega$ (see [7]). Both of these results generalize the classical smoothness properties of $\Omega$ (see [15], page 39, equation (25)). However, it is not obvious how to use these to obtain the variation results, even assuming smoothness of $\Omega$.

Remark 1.7. The oscillation as defined above, using the $\ell^{2}$-norm, dominates the corresponding operator defined using the $q$-norm for $q>2$. Thus there is no need to mention oscillation with $\ell^{q}$ norms, $q>2$. For $q<2$ the $q$-oscillation operator fails to be a bounded operator on any $L^{p}$ (with $I_{i}=\left[2^{i-1}, 2^{i}\right)$ ). See [1] for the case of differentiation operators. The argument presented there can be applied to the operators considered here as well.

Remark 1.8. In general, to obtain a variation result, we will need to assume $\varrho>$ 2. This already occurs in the case of martingales (see [13]) and in the case of differentiation operators.

Remark 1.9. The inequalities in Theorem $\mathrm{A}$ are not a consequence of a general convergence principle such as the Banach principle. A simple example of a family of operators that converges almost everywhere, but for which the oscillation and variation are identically $\infty$, is given in ([4, Remark 1.6).

We prove Theorem $\AA$ by deriving the type $(p, p)$ estimates first for odd kernels, then for even kernels. Since any kernel $K$ satisfying the hypotheses of Theorem A admits a decomposition $K(x)=K_{1}(x)+K_{2}(x)$, where $K_{1}$ is even, $K_{2}$ is odd, and each satisfies the hypotheses of the theorem, the type $(p, p)$ statement in Theorem A follows.

The treatment of odd kernels uses the method of rotations, which allows us to directly apply the one-dimensional results from [4]. For the even kernel, we transform it into an odd kernel by applying the vector-valued Riesz transform, and then use the one-dimensional techniques developed for convolution operators in [4] to handle error terms.

Because several results from [4] are referred to in these proofs, we have collected them in the Appendix, Section 6 .

The main tools in the proof of Theorem Bare the Calderón-Zygmund decomposition of an integrable function and an almost orthogonality lemma (Lemma 4.1). A key step is to organize the intervals of the partition $\left\{I_{i}\right\}$ into long and short components. Here, the long components contain a union of dyadic intervals, i.e., an interval of the form $\left[2^{k}, 2^{j}\right)$, and the short components are those contained in a single dyadic interval $\left[2^{i}, 2^{i+1}\right)$.

It is useful to introduce some alternative notation for the variation and oscillation of a one-parameter family of operators. If $\left\{W_{\epsilon}\right\}_{\epsilon>0}$ is such a family of operators, then $O(W f)$ and $V_{\varrho}(W f)$ are each semi-norms on the family $W_{\epsilon} f(x)$. We denote $O(W f)(x)$ by $\left\|W_{*} f(x)\right\|_{O}$ and $V_{\varrho}(W f)(x)$ by $\left\|W_{*} f(x)\right\|_{v_{\varrho}}$. See 9 for further discussion of these semi-norms.

That is,

$$
O(W f)(x)=\left\|W_{*} f(x)\right\|_{O}=\left(\sum_{i=1}^{\infty} \sup _{t_{i+1} \leq \epsilon_{i+1}<\epsilon_{i} \leq t_{i}}\left|W_{\epsilon_{i}} f(x)-W_{\epsilon_{i+1}} f(x)\right|^{2}\right)^{\frac{1}{2}},
$$


where $\left(t_{i}\right)$ is a fixed decreasing sequence, and

$$
V_{\varrho}(W f)(x)=\left\|W_{*} f(x)\right\|_{v_{\varrho}}=\sup _{\left(\epsilon_{i}\right) \backslash 0}\left(\sum_{i=1}^{\infty}\left|W_{\epsilon_{i}} f(x)-W_{\epsilon_{i+1}} f(x)\right|^{\varrho}\right)^{\frac{1}{\varrho}} .
$$

We may apply the above results on oscillation and variation to provide estimates on the $\lambda$-jump operator; that is,

$$
\begin{aligned}
& \Lambda\left(W_{\epsilon}, f, \lambda, x\right)=\sup \left\{n \geq 0: \text { there exist } s_{1}<t_{1} \leq s_{2}<t_{2}<\cdots \leq s_{n}<t_{n}\right. \\
& \text { with the property that } \left.\left|W_{t_{i}} f(x)-W_{s_{i}} f(x)\right|>\lambda, i=1,2, \ldots, n\right\},
\end{aligned}
$$

which gives the number of $\lambda$-jumps of the family $W_{\epsilon} f$. Clearly, if $\lim _{\epsilon \backslash 0} W_{\epsilon} f(x)$ exists a.e., then the number of $\lambda$-jumps must be finite a.e. The size of $\Lambda\left(W_{\epsilon}, f, \lambda, x\right)$ gives us information about how the family $W_{\epsilon} f(x)$ converges. For $K_{\epsilon} f(x)=$ $K_{[\epsilon, \infty)} f(x)$, we prove the following:

Theorem C. If $\varrho>2$, then the operator $\Lambda\left(K_{\epsilon}, f, \lambda, x\right)$ satisfies $\left\|\Lambda\left(K_{\epsilon}, f, \lambda, \cdot\right)^{\frac{1}{\varrho}}\right\|_{p} \leq$ $c(p, \varrho)\|f\|_{p}$ for $1<p<\infty$, and if $n \geq 1$ we have

$$
m\left\{x: \Lambda\left(K_{\epsilon}, f, \lambda, x\right)>n\right\} \leq \frac{c(\varrho)}{\lambda n^{\frac{1}{\varrho}}}\|f\|_{1} .
$$

Notation 1.10. Throughout the paper, $c$ and $C$, sometimes with additional parameters, will denote constants, but may not always denote the same constant from one occurrence to the next.

\section{Type $(p, p)$ ESTIMATES FOR OdD KERNELS}

In this section we prove that when the kernel $K$ is odd, the variation operator $f \rightarrow V_{\varrho}(K f)$, for $\varrho>2$, is type $(p, p), p>1$. The proof for the oscillation operator is similar and is therefore omitted.

For odd kernels we may relax the $L \log ^{+} L$ condition on $K$ in Theorem A.

Theorem 2.1. Let $K$ denote an odd kernel that is homogeneous of degree $-d$, and integrable on $\Sigma_{d-1}$. Then for $1<p<\infty$ we have

$$
\left\|V_{\varrho}(K f)(x)\right\|_{p} \leq c(p, \varrho)\|f\|_{p}, \quad \varrho>2,
$$

and

$$
\|O(K f)(x)\|_{p} \leq c(p)\|f\|_{p} .
$$

Proof. The hypotheses imply that $K(t)=\frac{\Omega(t)}{|t|^{d}}$, where $\Omega$ is odd, homogeneous of degree 0 , and in $L^{1}\left(\Sigma_{d-1}\right)$.

Let

$$
H_{I}^{1} f(x)=\int_{|s| \in I} \frac{f(x-s \cdot \mathbf{1})}{s} d s,
$$

and let $\left(R_{\sigma} f\right)(x)=f(\sigma x)$, where $\mathbf{1}=(1,0, \ldots, 0)$ is the first coordinate vector in $\mathbb{R}^{d}$, and $\sigma$ is a rotation on $\mathbb{R}^{d}$.

Let $t^{\prime}=t /|t| \in \Sigma_{d-1}$, and let $d \sigma$ denote Haar measure on $S O(d)$, normalized so that $\int_{S O(d)} d \sigma=\left|\Sigma_{d-1}\right|$, the Lebesgue measure of $\Sigma_{d-1}$. Following Stein and Weiss ([17], page 222), we have

$$
\int_{|t| \in I} f(x-t) \frac{\Omega\left(t^{\prime}\right)}{|t|^{d}} d t=\frac{1}{2} \int_{S O(d)}\left(R_{\sigma^{-1}} H_{I}^{1} R_{\sigma} f\right)(x) \Omega(\sigma \mathbf{1}) d \sigma .
$$


Thus, for any measurable choice of partitions $\left\{I_{i}\right\}=\left\{I_{i}(x)\right\}$ we have

$$
\begin{aligned}
& \left(\sum_{i}\left|\frac{1}{2} \int_{S O(d)}\left(R_{\sigma^{-1}} H_{I_{i}}^{1} R_{\sigma} f\right)(x) \Omega(\sigma \mathbf{1}) d \sigma\right|^{\varrho}\right)^{\frac{1}{\varrho}} \\
& \leq \frac{1}{2} \int_{S O(d)}\left(\sum_{i}\left|\left(R_{\sigma^{-1}} H_{I_{i}}^{1} R_{\sigma} f\right)(x)\right|^{\varrho}\right)^{\frac{1}{\varrho}}|\Omega(\sigma \mathbf{1})| d \sigma \\
& \leq \frac{1}{2} \int_{S O(d)} R_{\sigma^{-1}}\left(\sum_{i}\left|\left(H_{I_{i}}^{1} R_{\sigma} f\right)(x)\right|^{\varrho}\right)^{\frac{1}{\varrho}}|\Omega(\sigma \mathbf{1})| d \sigma \\
& \leq \frac{1}{2} \int_{S O(d)} R_{\sigma^{-1}} V_{\varrho}\left(H^{1}\left(R_{\sigma} f\right)\right)(x)|\Omega(\sigma \mathbf{1})| d \sigma .
\end{aligned}
$$

We now have, for $1<p<\infty$,

$$
\begin{aligned}
\left\|V_{\varrho}(K f)\right\|_{p} & \leq\left(\int_{\mathbb{R}^{d}}\left|\frac{1}{2} \int_{S O(d)} R_{\sigma^{-1}} V_{\varrho}\left(H^{1}\left(R_{\sigma} f\right)\right)(x)\right| \Omega(\sigma \mathbf{1})|d \sigma|^{p} d x\right)^{\frac{1}{p}} \\
& \leq \frac{1}{2} \int_{S O(d)}\left(\int_{\mathbb{R}^{d}}\left|R_{\sigma^{-1}} V_{\varrho}\left(H^{1}\left(R_{\sigma} f\right)\right)(x) \Omega(\sigma \mathbf{1})\right|^{p} d x\right)^{\frac{1}{p}} d \sigma \\
& \leq c(\varrho, p) \int_{S O(d)}\left\|R_{\sigma} f\right\|_{p}|\Omega(\sigma \mathbf{1})| d \sigma \\
& \leq c(\Omega, \varrho, p)\|f\|_{p} .
\end{aligned}
$$

\section{Type $(p, p)$ estimates FOR EVEn KeRnels}

In this section we prove that when the kernel $K$ is even, the variation operator $f \rightarrow V_{\varrho}(K f)$, for $\varrho>2$, is type $(p, p), p>1$.

Theorem 3.1. Let $K$ be a function on $\mathbb{R}^{d}$ with the properties

(1) $K$ is homogeneous of degree $-d$,

(2) $K$ is even,

(3) $K \log ^{+} K$ is integrable on the sphere $\Sigma=\{|x|=1\}$, and

(4) $\int_{\Sigma} K(x) d S(x)=0$.

Then $f \rightarrow V_{\varrho}(K f)(\varrho>2)$, and $f \rightarrow O(K f)$ are type $(p, p)$, for $1<p<\infty$.

In our proof we use the technique of Calderón and Zygmund in 3], which consists of "transforming" the even kernel into an odd kernel and then applying Theorem 2.1 .

Proof. Let $\vec{R}$ denote the vector-valued Riesz kernel on $\mathbb{R}^{d}$. That is,

$$
\vec{R}(x)=\frac{\Gamma\left(\frac{d+1}{2}\right)}{\pi^{(d+1) / 2}} \frac{x}{|x|^{d+1}} .
$$

This kernel is odd and homogeneous of degree $-d$. For $f \in L^{p}, 1<p<\infty$, let

$$
\vec{g}(x)=\vec{R} f(x)=\lim _{\epsilon \rightarrow 0} \int_{|x-y|>\epsilon} \vec{R}(x-y) f(y) d y .
$$

It is well known that the required limit exists a.e. and in $L^{p}$. Further, each component $g_{i}$ of $\vec{g}=\left(g_{1}, g_{2}, \ldots, g_{d}\right)$ is in $L^{p}\left(\mathbb{R}^{d}\right)$, and satisfies $\left\|g_{i}\right\|_{p} \leq c\|f\|_{p}$. 
We also know that

$$
-\vec{R}(\vec{R} f)=-\vec{R} \cdot \vec{g}(x)=\lim _{\epsilon \rightarrow 0} \sum_{j=1}^{d}-\int_{|x-y|>\epsilon} R_{j}(x-y) g_{j}(y) d y=f(x) .
$$

With this representation of $f$, the idea is to form a new convolution kernel, putting one operator $\vec{R}$ with the given even kernel, obtaining an odd kernel, and applying it to $\vec{R} f$, which we know is a bounded operator. However, there are a number of details that must be handled.

Define

$$
\vec{N}(x)=\lim _{\delta \rightarrow 0} \int_{|x-y|>\delta} \vec{R}(x-y) K(y) d y .
$$

Then, each component of $\vec{N}$ is an odd kernel, homogeneous of degree $-d$, with a singularity at $x=0$.

Let $\Phi$ be a continuously twice differentiable function on $\mathbb{R}$ such that

(1) $\Phi(t)=0$ if $t<1 / 4$, and $\Phi(t)=1$ if $t>\frac{3}{4}$,

(2) $0 \leq \Phi(t) \leq 1$ for any $t$, and

(3) $\Phi^{\prime}$ and $\Phi^{\prime \prime}$ are both bounded.

Then

$$
\vec{M}(x)=\lim _{\delta \rightarrow 0} \int_{|x-y|>\delta} \vec{R}(x-y) K(y) \Phi(|y|) d y
$$

is a better behaved odd kernel (the singularity at $x=0$ has been removed), but it does not have the required homogeneity (unlike $\vec{N}$ ).

The properties of $M$ and $N$ are summarized in the following lemma.

Lemma 3.2 (Calderón-Zygmund, [3]). The vector-valued kernels $\vec{N}$ and $\vec{M}$ have the following properties:

(1) Let $N$ denote one of the components of $\vec{N}$; then

(a) $N$ is integrable on $\Sigma$,

(b) $N$ is odd (thus, in particular, $\int_{\Sigma} N d S=0$ ), and

(c) $N$ is homogeneous of degree $-d$.

(2) Let $M$ denote the component of $\vec{M}$ corresponding to $N$; then

(a) $M$ is odd,

(b) $|N(x)-M(x)| \leq A /|x|^{d+1}$ for $|x|>1$, and

(c) for $|x| \leq 1,|M(x)| \leq G(x)$, where $G$ is homogeneous of degree 0 and integrable on $\Sigma$.

To study the oscillation and variation of

$$
K_{\epsilon} f(x)=\int_{|x-y|>\epsilon} K(x-y) f(y) d y,
$$

we use the identity (see [3], equation (5.10))

$$
\int K(x-y) \Phi\left(\frac{|x-y|}{\epsilon}\right) f(y) d y=\frac{1}{\epsilon^{d}} \int \vec{M}\left(\frac{x-y}{\epsilon}\right) \cdot \vec{g}(y) d y .
$$

Remark 3.3. This identity is not trivial to prove. The idea is to first prove it in the case when each component of $\vec{g}$ is continuously differentiable and has compact support. Then a change of order of integration and a change of variables show that the two equations are equal in this special case. A series of approximation arguments is used to complete the proof. See [3] for details. 
Hence we have

$$
\begin{aligned}
K_{\epsilon} f(x) & =\int K(x-y) \Phi\left(\frac{|x-y|}{\epsilon}\right) f(y) d y-\int_{|x-y| \leq \epsilon} K(x-y) \Phi\left(\frac{|x-y|}{\epsilon}\right) f(y) d y \\
& =\frac{1}{\epsilon^{d}} \int \vec{M}\left(\frac{x-y}{\epsilon}\right) \cdot \vec{g}(y) d y-\int_{|x-y| \leq \epsilon} K(x-y) \Phi\left(\frac{|x-y|}{\epsilon}\right) f(y) d y \\
& =\vec{M}_{\epsilon} * \vec{g}(x)-\tilde{K}_{\epsilon} * f(x),
\end{aligned}
$$

where for each $j, 1 \leq j \leq d$, the $j$ th component, $g_{j}$, satisfies $g_{j} \in L^{p},\left\|g_{j}\right\|_{p} \leq$ $C\|f\|_{p}(1<p<\infty)$, and

$$
\vec{M}_{\epsilon}(x)=\frac{1}{\epsilon^{d}} \vec{M}\left(\frac{x}{\epsilon}\right) \quad \text { and } \quad \tilde{K}_{\epsilon}(x)=K(x) \Phi\left(\frac{|x|}{\epsilon}\right) \chi_{[0, \epsilon]}(|x|) .
$$

We further decompose each component of the kernel $\vec{M}$. In what follows, to simplify the notation, we will work with a single component of each of the vectors involved, and suppress the associated subscripts.

Write

$$
\begin{aligned}
\frac{1}{\epsilon^{d}} \int & M\left(\frac{x-y}{\epsilon}\right) g(y) d y \\
= & \int_{|x-y|>\epsilon} N(x-y) g(y) d y+\frac{1}{\epsilon^{d}} \int_{|x-y| \leq \epsilon} M\left(\frac{x-y}{\epsilon}\right) g(y) d y \\
& +\frac{1}{\epsilon^{d}} \int_{|x-y|>\epsilon}\left[M\left(\frac{x-y}{\epsilon}\right)-N\left(\frac{x-y}{\epsilon}\right)\right] g(y) d y \\
= & N_{\epsilon} g(x)+\tilde{M}_{\epsilon} g(x)+D_{\epsilon} g(x),
\end{aligned}
$$

where

$$
\tilde{M}_{\epsilon}(y)=\frac{1}{\epsilon^{d}} M\left(\frac{y}{\epsilon}\right) \chi_{(0, \epsilon)}(y) \text { and } D_{\epsilon}(y)=\frac{1}{\epsilon^{d}}\left(M\left(\frac{y}{\epsilon}\right)-N\left(\frac{y}{\epsilon}\right) \chi_{(0, \epsilon)}(y)\right) .
$$

Therefore to study $\left\|K_{*} f(x)\right\|_{v_{\varrho}}$, it will be enough to study

$$
\left\|N_{*} g(x)+\tilde{M}_{*} g(x)+D_{*} g(x)-\tilde{K}_{*} f(x)\right\|_{v_{\varrho}} .
$$

Hence by the triangle inequality, it will be enough to study

$$
\left\|N_{*} g(x)\right\|_{v_{\varrho}}, \quad\left\|\tilde{M}_{*} g(x)\right\|_{v_{\varrho}}, \quad\left\|D_{*} g(x)\right\|_{v_{\varrho}}, \quad\left\|\tilde{K}_{*} f(x)\right\|_{v_{\varrho}} .
$$

The same considerations apply if we want to study the oscillation operator. Since there are no additional complications, we will only consider the variation operator.

We will make one other decomposition before we continue. Write

$$
\tilde{M}(y)=N(y) \Phi(|y|) \chi_{\{|y| \leq 1\}}+[M(y)-N(y) \Phi(|y|)] \chi_{\{|y| \leq 1\}}=N^{\Phi}(y)+\Delta(y) \text {. }
$$

Therefore,

$$
\begin{aligned}
\tilde{M}_{\epsilon} g(x) & =\frac{1}{\epsilon^{d}} \int_{|x-y| \leq \epsilon} M\left(\frac{x-y}{\epsilon}\right) g(y) d y \\
& =\frac{1}{\epsilon^{d}} \int_{|x-y| \leq \epsilon} N^{\Phi}\left(\frac{x-y}{\epsilon}\right) g(y) d y+\frac{1}{\epsilon^{d}} \int_{|x-y| \leq \epsilon} \Delta\left(\frac{x-y}{\epsilon}\right) g(y) d y .
\end{aligned}
$$

By the properties of the kernel $N$ and Theorem 2.1 we have the estimate \|\|$N_{*} g(x)\left\|_{v_{\varrho}}\right\|_{p} \leq c(p, \varrho)\|f\|_{p}$, for $\varrho>2$ and $1<p<\infty$. Similarly, a good estimate holds for the oscillation of this piece. 
For the other kernels we will reduce the situation to a one-dimensional problem, and employ the lemmas in section 6.

Lemma 3.4. Let $T$ be a kernel on $\mathbb{R}^{d}$ that is integrable on the sphere $\Sigma$. For each $u$ with $|u|=1$, let $\varphi_{u}$ have support on $[0, \infty)$ and satisfy

$$
T_{\epsilon} f(x)=\int_{\Sigma} T(u) \int_{0}^{\infty} \varphi_{u}(t) f(x-\epsilon t u) d t d S(u) .
$$

If $O\left(\varphi_{u} * h\right)$ and $V_{\varrho}\left(\varphi_{u} * h\right)$ with $\varrho>2$ are type $(p, p)$ for $1<p<\infty$, for functions $h: \mathbb{R} \rightarrow \mathbb{R}$, and with a bound independent of $u$, then $O(T f)$ and $V_{\varrho}(T f)$ with $\varrho>2$ are type $(p, p)$ for $1<p<\infty$ for functions $f: \mathbb{R}^{d} \rightarrow \mathbb{R}$.

Proof. The proof follows the lines of the case for odd kernels given earlier.

Note that

$$
\begin{aligned}
\left\|T_{*} f(x)\right\|_{v_{\varrho}} & =\sup _{\left(\epsilon_{i}\right)}\left(\sum_{i=1}^{\infty}\left|T_{\epsilon_{i}} f(x)-T_{\epsilon_{i+1}} f(x)\right|^{\varrho}\right)^{\frac{1}{\varrho}} \\
= & \sup _{\left(\epsilon_{i}\right)}\left(\sum_{i=1}^{\infty} \mid \int_{\Sigma_{d-1}} T\left(t^{\prime}\right)\left(\int_{0}^{\infty} \varphi_{u}(r) f\left(x-t^{\prime} \epsilon_{i} r\right) d r\right.\right. \\
& \left.\left.-\int_{0}^{\infty} \varphi_{u}(r) f\left(x-t^{\prime} \epsilon_{i+1} r\right) d r\right)\left.d S\left(t^{\prime}\right)\right|^{\varrho}\right)^{\frac{1}{\varrho}} \\
\leq & \sup _{\left(\epsilon_{i}\right)} \int_{\Sigma_{d-1}}\left|T\left(t^{\prime}\right)\right|\left(\sum_{i=1}^{\infty} \mid \int_{0}^{\infty} \varphi_{u}(r) f\left(x-t^{\prime} \epsilon_{i} r\right) d r\right. \\
\leq & -\int_{\Sigma_{d-1}}^{\infty}\left|T\left(t^{\prime}\right)\right|\left\|T_{*}^{t^{\prime}, u} f(x)\right\|_{v_{\varrho}} d S\left(t^{\prime}\right),
\end{aligned}
$$

where

$$
T_{\epsilon}^{t^{\prime}, u} f(x)=\int_{0}^{\infty} \varphi_{u}(r) f\left(x-t^{\prime} \epsilon r\right) d r .
$$

Since we already have the variational inequality for the one-dimensional convolution with $\varphi$, we can obtain the same thing in the above case.

For $1<p<\infty$ we have

$$
\begin{aligned}
\|\| T_{*} f\left\|_{v_{\varrho}}\right\|_{p} & \leq\left(\int_{R^{d}}\left|\int_{\Sigma_{d-1}}\right| T\left(t^{\prime}\right)\left|\left\|T_{*}^{t^{\prime}} f(x)\right\|_{v_{\varrho}} d S\left(t^{\prime}\right)\right|^{p} d x\right)^{\frac{1}{p}} \\
& \leq \int_{\Sigma_{d-1}}\left|T\left(t^{\prime}\right)\right|\left(\int_{R^{d}}\left\|T_{*}^{t^{\prime}, u} f(x)\right\|_{v_{\varrho}}^{p} d x\right)^{\frac{1}{p}} d S\left(t^{\prime}\right) \\
& \leq c(T, \varrho, p)\|f\|_{p} .
\end{aligned}
$$

The same argument also shows the result for $O(T f)$.

We will now show that each of the kernels $\tilde{K}, N^{\Phi}, \Delta$ and $D$ has a representation that satisfies the hypothesis of Lemma 3.4 
The $\tilde{K}$ kernel:

$$
\begin{aligned}
\int_{|x-y| \leq \epsilon} K(x-y) \Phi\left(\frac{|x-y|}{\epsilon}\right) f(y) d y & =\int_{|y| \leq 1} K(y) \Phi(|y|) f(x-\epsilon y) d y \\
& =\int_{\Sigma} K(u) \int_{0}^{1} \frac{\Phi(t)}{t} f(x-\epsilon t u) d t d S(u) .
\end{aligned}
$$

Now $\varphi(t)=\frac{\Phi(t)}{t} \chi_{[1 / 4,1]}(t)$ satisfies the hypothesis of Lemma 6.4 (i.e., a prior 1dimensional estimate; see the Appendix). Hence $\tilde{K}$ satisfies the hypothesis of Lemma 3.4 .

The $N_{\epsilon}^{\Phi}$ kernel:

$$
\begin{aligned}
N_{\epsilon}^{\Phi} g(x) & =\int_{|y| \leq 1} \Phi(|y|) N(y) g(x-\epsilon y) d y \\
& =\int_{\Sigma} N(u) \int_{1 / 4}^{1} \frac{\Phi(t)}{t} g(x-\epsilon t u) d t d S(u) \\
& =\int_{\Sigma} N(u) \int_{1 / 4}^{1} \psi(t) g(x-\epsilon t u) d t d S(u),
\end{aligned}
$$

where $\psi(t)=\frac{\Phi(t)}{t} \chi_{[1 / 4,1]}(t)$. Since $\psi(t)$ is differentiable, $\int_{0}^{1} t\left|\psi^{\prime}(t)\right| d t<\infty$, and $\int_{\Sigma}|N| d S<\infty$, then $\tilde{N}$ satisfies the hypothesis of Lemma 3.4

The $\Delta$ kernel:

$$
\Delta_{\epsilon} g(x)=\int_{|y| \leq 1} \Delta(y) g(x-\epsilon y) d y=\int_{\Sigma} \int_{0}^{1} t^{d-1} \Delta(t u) g(x-\epsilon t u) d t d S(u) .
$$

Since $\int_{\Sigma} K d S=0$, for each $t, 0<t \leq 1$, we have

$$
\begin{aligned}
\Delta(y) & =\int R(y-z) K(z)[\Phi(|z|)-\Phi(|y|)] d z \\
& =\int\left[R(y-z)-\chi_{|z|<t} R(y)\right] K(z)[\Phi(|z|)-\Phi(|y|)] d z .
\end{aligned}
$$

Since $R$ and $K$ are both homogeneous of degree $-d$,

$$
\Delta(t u)=\int\left[R(t u-z)-\chi_{|z|<t} R(t u)\right] K(z)[\Phi(|z|)-\Phi(|t u|)] d z .
$$

Using this, and making the change $z=t w$, we have

$$
\begin{aligned}
\Delta(t u) & =\int\left[R(t u-t w)-\chi_{|t w|<t} R(t u)\right] K(t w)[\Phi(|t w|)-\Phi(|t u|)] t^{d} d w \\
& =\int \frac{1}{t^{d}}\left[R(u-w)-\chi_{|w|<1} R(u)\right] \frac{1}{t^{d}} K(w)[\Phi(|t w|)-\Phi(|t u|)] t^{d} d w \\
& =\int\left[R(u-w)-\chi_{|w|<1} R(u)\right] K(w) \frac{\Phi(|t w|)-\Phi(|t u|)}{t^{d}} d w .
\end{aligned}
$$

Then

$$
\begin{aligned}
\varphi_{u}(t) & =t^{d-1} \Delta(t u) \chi_{[0,1]}(t) \\
& =\int\left[R(u-w)-\chi_{|w|<1} R(u)\right] K(w) \frac{\Phi(t|w|)-\Phi(t|u|)}{t} d w \chi_{[0,1]}(t) .
\end{aligned}
$$

By Lemmas 6.4 and 3.4 all we need to show is that $\int_{0}^{1} t\left|\frac{d \varphi_{u}(t)}{d t}\right| d t \leq A<\infty$. 
Recalling that $|u|=1$, we have to show that

$$
\begin{aligned}
& \text { (3.2) } \int_{0}^{1} t\left|\frac{d \varphi_{u}(t)}{d t}\right| d t \\
& =\int_{0}^{1} t \int\left|R(u-w)-\chi_{|w|<1} R(u)\right||K(w)|\left|\frac{d}{d t} \frac{\Phi(t|w|)-\Phi(t)}{t}\right| d w d t \leq A<\infty .
\end{aligned}
$$

We will need the estimate (see [3], page 301), valid if $1 / 8<|u| \leq 1$,

$$
\left|R(u-w)-\chi_{[|w|<1]} R(u)\right| \leq c \frac{|w|^{1 / 2}}{|u-w|^{d}} .
$$

We will use this estimate with $|u|=1$. Split the domain of integration for $w$ into three regions:

$$
\begin{aligned}
& D_{1}=\left\{w:|w|>\frac{2}{t}\right\} \\
& D_{2}=\left\{w:|w| \leq \frac{2}{t}\right\} \cap\{w:|u-w|>.1\} ; \\
& D_{3}=\left\{w:|w| \leq \frac{2}{t}\right\} \cap\{w:|u-w| \leq .1\} .
\end{aligned}
$$

In the region $D_{1}$, since $0<t<1$, we have $|w|>2$, so that $|u-w|>c w$. Using this, we have the estimate

$$
\left|R(u-w)-\chi_{[|w|<1]} R(u)\right|=|R(u-w)| \leq \frac{c}{|u-w|^{d}} \leq \frac{c}{|w|^{d}} .
$$

Further, in this region, $\Phi(t|w|)=1$, and we see that

$$
\left|\frac{d}{d t} \frac{\Phi(t|w|)-\Phi(t)}{t}\right| \leq \frac{c}{t^{2}}
$$

With these estimates we see that the integral in (3.2), over the region $D_{1}$, is dominated by

$$
\begin{aligned}
& \int_{0}^{1} t \int_{w \in D_{1}} \frac{c}{|w|^{d}}|K(w)| \frac{1}{t^{2}} d w d t \\
& =\int_{|w|>2}\left(\int_{\frac{2}{|w|}}^{1} \frac{1}{t} d t\right) \frac{c}{|w|^{d}}|K(w)| d w \\
& =\int_{|w|>2} \ln \left(\frac{|w|}{2}\right) \cdot \frac{c}{|w|^{d}}|K(w)| d w,
\end{aligned}
$$

and this is finite.

Now to $D_{2}$. We begin by observing that

$$
\begin{array}{r}
\left|\frac{d}{d t} \frac{\Phi(t|w|)-\Phi(t)}{t}\right|=\left|\frac{\left[\Phi^{\prime}(t|w|)|w|-\Phi^{\prime}(t)\right] t-[\Phi(t|w|)-\Phi(t)]}{t^{2}}\right| \\
\leq\left|\frac{\Phi^{\prime}(t|w|)|w|-\Phi^{\prime}(t)}{t}\right|+\left|\frac{\Phi(t|w|)-\Phi(t|u|)}{t^{2}}\right|
\end{array}
$$


Using the mean value theorem, we may estimate the numerator in the left-most term on the last line of (3.4) by

$$
\begin{aligned}
\left|\Phi^{\prime}(t|w|)\right| w\left|-\Phi^{\prime}(t)\right| & =\left|\left(\Phi^{\prime}(t|w|)|w|-\Phi^{\prime}(t)|w|\right)+\left(\Phi^{\prime}(t)|w|-\Phi^{\prime}(t)\right)\right| \\
& \leq\left|\Phi^{\prime}(t|w|)-\Phi^{\prime}(t)\right||w|+\left|\Phi^{\prime}(t)\right||| w|-1| \\
& \leq\left|\Phi^{\prime \prime}(s)\right||t| w|-t||w|+\left|\Phi^{\prime}(t)\right||| w|-1| \\
& \leq\left|\Phi^{\prime \prime}(s)\right| t|w||| w|-1|+\left|\Phi^{\prime}(t)\right||| w|-1| \\
& \leq\left(\left|\Phi^{\prime \prime}(s)\right| t|w|+\left|\Phi^{\prime}(t)\right|\right)|| w|-1| .
\end{aligned}
$$

In this region, $t|w| \leq 2$, and $\Phi^{\prime}$ and $\Phi^{\prime \prime}$ are bounded. So we may estimate the above by

$$
C|| w|-1|=C|| w|-| u|| \leq C|w-u| .
$$

The right-most term in the last line of (3.4) may be estimated by

$$
\left|\frac{\Phi(t|w|)-\Phi(t|u|)}{t^{2}}\right| \leq\left|\frac{\Phi^{\prime}(v)(|w|-|u|) t}{t^{2}}\right| \leq C \frac{|w-u|}{t},
$$

and, combining this with the previous estimate for the left-most term, we see that

$$
\left|\frac{d}{d t} \frac{\Phi(t|w|)-\Phi(t)}{t}\right| \leq C \frac{|w-u|}{t}
$$

Using these estimates, we see that the integral in (3.2), over the region $D_{2}$, may be estimated by

$$
\begin{aligned}
& \int_{0}^{1} t \int_{w \in D_{2}} \frac{\sqrt{|w|}}{|u-w|^{d}}|K(w)| c \frac{|u-w|}{t} d w d t \\
& \quad \leq \int_{0}^{1} \int_{|w|<2} C \sqrt{|w|}|K(w)| d w d t+\int_{0}^{1} \int_{|w|>2} C \frac{\sqrt{|w|}}{|w|^{d-1}}|K(w)| d w d t
\end{aligned}
$$

and both of these integrals are finite.

For the region $D_{3}$ we have that $.9<|w|<1.1$. Combined with the estimate in (3.3), this yields

$$
\left|R(u-w)-\chi_{[|w|<1]} R(u)\right| \leq c \frac{\sqrt{|w|}}{|u-w|^{d}} \leq c \frac{1}{|u-w|^{d}}, \quad w \in D_{3} .
$$

The estimate in (3.5) also holds in $D_{3}$. Thus we may estimate the integral in (3.2) over the region $D_{3}$ by

$$
\begin{aligned}
& \int_{0}^{1} t \int_{w \in D_{3}} \frac{\sqrt{|w|}}{|u-w|^{d}}|K(w)| c \frac{|u-w|}{t} d w d t \\
& \leq \int_{0}^{1} \int_{w \in D_{3}} C \frac{|K(w)|}{|w-u|^{d-1}} d w d t \\
& \leq \int_{0}^{1} \int_{9<|w|<1.1} C \frac{|K(w)|}{|w-u|^{d-1}} d w d t
\end{aligned}
$$

which has an integrable singularity; so this piece is bounded as well. 
The $D_{\epsilon}$ kernel:

$$
\begin{aligned}
D_{\epsilon} g(x) & =\epsilon^{-d} \int_{|x-y|>\epsilon} D\left(\frac{x-y}{\epsilon}\right) g(y) d y \\
& =\int_{|y|>1} D(y) g(x-\epsilon y) d y \\
& =\int_{\Sigma} \int_{1}^{\infty} t^{d-1} D(t u) g(x-\epsilon t u) d t d S(u) .
\end{aligned}
$$

For $|x| \geq 1$ we have $D(x)=M(x)-N(x)=M(x)-\Phi(|x|) N(x)$. Note that for $|t z|>3 / 4$ we have $\Phi(|t z|)=1$, and for $t>1$ we have $\Phi(t|u|)=1$. Hence, for $t \geq 1$,

$$
\begin{aligned}
\varphi_{u}(t) & =t^{d-1} D(t u) \chi_{[1, \infty)}(t) \\
& =\int R(u-z) K(z) \frac{[\Phi(t|z|)-\Phi(|t u|)]}{t} d z \\
& =\int_{|t z|<3 / 4}[R(u-z)-R(u)] K(z) \frac{[\Phi(t|z|)-\Phi(|t u|)]}{t} d z .
\end{aligned}
$$

By Lemmas 6.3 and 3.4 we need to show that

$$
\int_{1}^{\infty} t\left|\frac{d \varphi_{u}(t)}{d t}\right| d t<A<\infty
$$

for some constant $A$.

We have

$$
\left|\frac{d}{d t} \frac{[\Phi(t|z|)-\Phi(|t u|)]}{t}\right|=\left|\frac{|z| \Phi^{\prime}(|z| t) t-\Phi(t|z|)+1}{t^{2}}\right| \leq \frac{c}{t^{2}} .
$$

This, together with equation (3.3), gives

$$
\begin{aligned}
& \int_{1}^{\infty} t\left|\frac{d \varphi_{u}(t)}{d t}\right| d t \\
& \leq c \int_{1}^{\infty} \int_{|t z|<3 / 4}|R(u-z)-R(u)||K(z)| \frac{c}{t} d z d t \\
& \leq c \int_{1}^{\infty} \int_{|t z|<3 / 4} \frac{|z|^{1 / 2}}{|u-z|^{d}} \frac{|\Omega(z)|}{|z|^{d}} \frac{1}{t} d z d t \\
& \leq \int_{|z|<3 / 4} \frac{|z|^{1 / 2}}{|u-z|^{d}} \frac{|\Omega(z)|}{|z|^{d}} \int_{1}^{\frac{3}{4 z}} \frac{1}{t} d t d z \\
& \leq \int_{|z|<3 / 4}|\Omega(z)| \frac{c}{|z|^{d-1 / 2}} c \ln \frac{1}{|z|} d z=A<\infty .
\end{aligned}
$$

This concludes the proof of Theorem 3.1

\section{Proof that type $(2,2)$ implies Weak-type $(1,1)$}

In this section we prove Theorem B During the proof we refer to the following "almost orthogonality lemma". Lemmas of this type originate in the work of Cotlar ([5]), with further development due to Stein (see for example [11]). 
Lemma 4.1. Let $\left(d_{n}\right)$ be a sequence and $\left(h_{k, n}\right)$ be a double sequence of vectors in a normed space $(B,\|\cdot\|)$. Let $(\sigma(j))_{j \in \mathbb{Z}}$ be a sequence of positive numbers with $w=\sum_{j} \sigma(j)<\infty$. Suppose that

$$
\left\|h_{k, n}\right\| \leq \sigma(n-k)\left\|d_{n}\right\|
$$

for every $n, k$. Then

$$
\sum_{k}\left\|\sum_{n} h_{k, n}\right\|^{2} \leq w^{2} \cdot \sum_{n}\left\|d_{n}\right\|^{2} .
$$

A proof of the almost orthogonality lemma may be found in [10].

Proof of Theorem $B$. Let $\left\{I_{i}\right\}$ denote a collection of intervals which define $\mathcal{W}_{\varrho} f(x)$ $=\left(\sum_{i}\left|K_{I_{i}(x)} f(x)\right|^{\varrho}\right)^{1 / \varrho}$. From now on we suppose that this collection of intervals is fixed.

For every integer $n$, let the set $\mathbb{L}_{n}$ consist of all cubes of the form

$$
\left[s_{1} 2^{n-1},\left(s_{1}+1\right) 2^{n-1}\right) \times\left[s_{2} 2^{n-1},\left(s_{2}+1\right) 2^{n-1}\right) \times \cdots \times\left[s_{d} 2^{n-1},\left(s_{d}+1\right) 2^{n-1}\right),
$$

where $s_{1}, s_{2}, \ldots, s_{d}$ are integers; that is, $\mathbb{L}_{n}$ is the dyadic grid with cubes of side length $2^{n-1}$.

According to the Calderón-Zygmund decomposition (with $\alpha=1$ ) we can write $f=g+b$, where the functions $g, b$ have the following properties:

(1) $\|g\|_{L^{2}}^{2} \leq c \cdot\|f\|_{L^{1}}$.

(2) $b=\sum_{j} b_{j}$ where each $b_{j}$ satisfies:

(a) for some $n, b_{j}$ is supported in a cube $B_{j} \in \mathbb{L}_{n}$,

(b) $\int b_{j}=\int_{B_{j}} b_{j}=0$

(c) $\left\|b_{j}\right\|_{L^{1}} \leq c \cdot\left|B_{j}\right|$ and

(d) $\sum_{j}\left|B_{j}\right| \leq c \cdot\|f\|_{L^{1}}$.

In proving that the operator $\left(\sum_{i}\left|K_{I_{i}} f(x)\right|^{\varrho}\right)^{1 / \varrho}$ is weak $(1,1)$, we can assume that $\alpha=1$. Since this operator is subadditive, it is enough to prove that

$$
\begin{aligned}
& \left|\left\{\left(\sum_{i}\left|K_{I_{i}} g(x)\right|^{\varrho}\right)^{1 / \varrho}>1 / 2\right\}\right| \leq c \cdot\|f\|_{L^{1}\left(\mathbb{R}^{d}\right)}, \\
& \left|\left\{\left(\sum_{i}\left|K_{I_{i}} b(x)\right|^{\varrho}\right)^{1 / \varrho}>1 / 2\right\}\right| \leq c \cdot\|f\|_{L^{1}\left(\mathbb{R}^{d}\right)} .
\end{aligned}
$$

The inequality with $g$ follows from the assumption in equation (1.9) and from the estimate in property 1 of the Calderón-Zygmund decomposition.

Let us denote by $\tilde{B}_{j}$ the cube with the same center as $B_{j}$, but with each dimension expanded three times, and set $\tilde{B}=\bigcup_{j} \tilde{B}_{j}$. Because of the estimate in $2(\mathrm{~d})$ of the Calderón-Zygmund decomposition., it is enough to show that

$$
\left|\left\{x \in \tilde{B}^{c} \mid\left(\sum_{i}\left|K_{I_{i}} b(x)\right|^{\varrho}\right)^{1 / \varrho}>1 / 2\right\}\right| \leq c \cdot \sum_{j}\left|B_{j}\right| .
$$

For each $x \in \tilde{B}^{c}$ and $i, K_{I_{i}} b(x)$ can be nonzero only if, for some $j, B_{j}$ lies entirely in $A_{I_{i}}+x$ or $B_{j}$ intersects the boundary of $A_{I_{i}}+x$. Let $\partial A$ denote the boundary 
of a set $A$. We write

$$
\sum_{j}[\operatorname{Property}(j)] a_{j}
$$

to indicate the sum over $j$ for which $\operatorname{Property}(j)$ is true, of the $a_{j}$.

With these conventions, we have

$$
\begin{aligned}
\left(\sum_{i}\left|K_{I_{i}} b(x)\right|^{\varrho}\right)^{1 / \varrho}= & \left(\sum_{i}\left|\sum_{j} K_{I_{i}} b_{j}(x)\right|^{\varrho}\right)^{1 / \varrho} \\
\leq & \left(\sum_{i}\left|\sum_{j}\left[B_{j} \subset\left(A_{I_{i}}+x\right)\right] K_{I_{i}} b_{j}(x)\right|^{\varrho}\right)^{1 / \varrho} \\
& \quad+\left(\sum_{i}\left|\sum_{j}\left[B_{j} \cap\left(\partial A_{I_{i}}+x\right) \neq \varnothing\right] K_{I_{i}} b_{j}(x)\right|^{\varrho}\right)^{1 / \varrho} \\
= & \text { Interior Sum }+ \text { Boundary Sum. }
\end{aligned}
$$

It will be enough to show the following two inequalities:

$$
\begin{array}{r}
\mid\left\{x \in \tilde{B}^{c} \mid(\text { Interior Sum })>1 / 4\right\}\left|\leq c \cdot \sum_{j}\right| B_{j} \mid, \\
\mid\left\{x \in \tilde{B}^{c} \mid(\text { Boundary Sum })>1 / 4\right\}\left|\leq c \cdot \sum_{j}\right| B_{j} \mid .
\end{array}
$$

Let us first deal with the estimate for the interior sum, (4.2). We will prove that

$$
\int_{\tilde{B}^{c}}(\text { Interior Sum }) \leq c \cdot \sum_{j}\left|B_{j}\right|
$$

and since

$$
\mid\left\{x \in \tilde{B}^{c} \mid(\text { Interior Sum })>1 / 4\right\} \mid \leq 4 \int_{\tilde{B}^{c}} \text { (Interior Sum), }
$$

equation (4.2) will follow.

Since the $\ell^{\varrho}$ norm is not more than the $\ell^{1}$ norm, and since $b_{j}$ is supported on $B_{j} \subset A_{I_{i}}$, we have

$$
\begin{aligned}
\text { Interior Sum } & \leq \sum_{i}\left|\sum_{j}\left[B_{j} \subset\left(A_{I_{i}}+x\right)\right] K_{I_{i}} b_{j}(x)\right| \\
& \leq \sum_{j}\left|\int K(y-x) b_{j}(y) d y\right| .
\end{aligned}
$$

It follows that

$$
\int_{\tilde{B}^{c}} \text { Interior Sum } \leq \sum_{j} \int_{\tilde{B}_{j}^{c}}\left|\int K(y-x) b_{j}(y) d y\right| d x,
$$

and we just need to prove that, for each $j$,

$$
\int_{\tilde{B}_{j}^{c}}\left|\int K(y-x) b_{j}(y) d y\right| d x \leq c \cdot\left|B_{j}\right| .
$$

Let $y_{j}$ denote the center of $B_{j}$. By $2(\mathrm{~b})$, we have

$$
\int K(y-x) b_{j}(y) d y=\int\left(K(y-x)-K\left(y_{j}-x\right)\right) b_{j}(y) d y .
$$


Then from (1.8) and 2(c) we have

$$
\begin{aligned}
\int_{\tilde{B}_{j}^{c}}\left|\int K(y-x) b_{j}(y) d y\right| d x & \leq \int_{\tilde{B}_{j}^{c}} \int\left|K(y-x)-K\left(y_{j}-x\right)\right|\left|b_{j}(y)\right| d y d x \\
& =\int\left|b_{j}(y)\right| \int_{\tilde{B}_{j}^{c}}\left|K(y-x)-K\left(y_{j}-x\right)\right| d x d y \\
& \leq c \cdot \int\left|b_{j}(y)\right| d y \\
& \leq c \cdot\left|B_{j}\right|
\end{aligned}
$$

finishing the estimate on the interior sum (4.2).

Let us now estimate the boundary sum (4.3).

We start by subdividing some of the intervals in the collection $\left\{I_{i}\right\}$. Take an interval $I=[a, b)$ from $\left\{I_{i}\right\}$. If there are no integer powers of 2 in the interior of $I$, then we leave $I$ alone. If there is one integer power of 2 in the interior of $I$, say $2^{n}$, then divide $I$ into two parts as $\left[a, 2^{n}\right)$ and $\left[2^{n}, b\right)$. Suppose now that there are two or more powers of 2 in the interior of $I$. Then let $2^{n}$ be the smallest power of 2 , and let $2^{m}$ be the largest power of 2 in the interior of $I$, and divide $I$ into three intervals as $\left[a, 2^{n}\right),\left[2^{n}, 2^{m}\right)$ and $\left[2^{m}, b\right)$.

Note that by replacing the $\left\{I_{i}\right\}$ with this new collection of intervals, we increased the value of $\left(\sum_{i}\left|K_{I_{i}} f(x)\right|^{\varrho}\right)^{1 / \varrho}$ at most by a factor of $3^{1-1 / \varrho}$. Indeed, the worst case is when we divide $I$ into three parts, say $I=J_{1} \cup J_{2} \cup J_{3}$; in this case we have the estimate

$$
\begin{aligned}
\left|K_{I} f\right|^{\varrho} & =\left|K_{J_{1}} f+K_{J_{2}} f+K_{J_{3}} f\right|^{\varrho} \\
& \leq 3^{\varrho(1-1 / \varrho)} \cdot\left(\left|K_{J_{1}} f\right|^{\varrho}+\left|K_{J_{2}} f\right|^{\varrho}+\left|K_{J_{3}} f\right|^{\varrho}\right),
\end{aligned}
$$

using Hölder's inequality in the last step.

We now see that we can assume that the collection $\left\{I_{i}\right\}$ contains intervals $I_{i}$, which either satisfy $I_{i} \subset\left[2^{k}, 2^{k+1}\right)$ for some integer $k$, or $I_{i}$ is a dyadic interval, that is, its endpoints are (different) powers of 2. Correspondingly, for a given collection $\left\{I_{i}\right\}$, let us divide the indices $i$ into two sets according to $I_{i}$ being "short" or "long":

$$
\begin{aligned}
& \mathcal{S}=\left\{i \mid I_{i} \subset\left[2^{k}, 2^{k+1}\right) \text { for some integer } k\right\}, \\
& \mathcal{L}=\left\{i \mid I_{i} \text { is dyadic }\right\} .
\end{aligned}
$$

It will now be enough to prove the inequalities

$$
\begin{aligned}
& \left|\left\{\left(\sum_{i \in \mathcal{S}}\left|\sum_{j}\left[B_{j} \cap\left(\partial A_{I_{i}}+x\right) \neq \varnothing\right] K_{I_{i}} b_{j}(x)\right|^{\varrho}\right)^{1 / \varrho}>1 / 8\right\}\right| \leq c \cdot \sum_{j}\left|B_{j}\right|, \\
& \left|\left\{\left(\sum_{i \in \mathcal{L}}\left|\sum_{j}\left[B_{j} \cap\left(\partial A_{I_{i}}+x\right) \neq \varnothing\right] K_{I_{i}} b_{j}(x)\right|^{\varrho}\right)^{1 / \varrho}>1 / 8\right\}\right| \leq c \cdot \sum_{j}\left|B_{j}\right| .
\end{aligned}
$$

We will refer to the inequality in (4.4) as the short inequality, and we will call (4.5) the long inequality.

The proof of the long inequality, (4.5), is straightforward; so let us prove it first. 
Denote by $2^{\beta_{j}}$ the side length of $B_{j}$, and let $J_{k}$ denote the interval $\left[2^{k}, 2^{k+1}\right)$. Since the $\ell^{\varrho}$ norm is no more than the $\ell^{1}$ norm, we can estimate

$$
\begin{aligned}
& \left(\sum_{i \in \mathcal{L}}\left|\sum_{j}\left[B_{j} \cap\left(\partial A_{I_{i}}+x\right) \neq \varnothing\right] K_{I_{i}} b_{j}(x)\right|^{\varrho}\right)^{1 / \varrho} \\
& \quad \leq \sum_{i \in \mathcal{L}}\left|\sum_{j}\left[B_{j} \cap\left(\partial A_{I_{i}}+x\right) \neq \varnothing\right] K_{I_{i}} b_{j}(x)\right| \\
& \quad \leq \sum_{k \in \mathbb{Z}}\left|\sum_{j}\left[B_{j} \cap\left(\partial A_{J_{k}}+x\right) \neq \varnothing\right] K_{J_{k}} b_{j}(x)\right| \\
& \quad \leq \sum_{j} \sum_{k>\beta_{j}}\left[B_{j} \cap\left(\partial A_{J_{k}}+x\right)\right] \int_{A_{J_{k}}}|K(y)|\left|b_{j}(x+y)\right| d y .
\end{aligned}
$$

For each $k, j$, define

$$
F_{j, k}=\left\{x \in \tilde{B}^{c} \mid B_{j} \cap\left(x+\partial A_{J_{k}}\right) \neq \varnothing\right\} .
$$

We need to prove that

$$
\sum_{j} \sum_{k>\beta_{j}} \int_{F_{j, k}} \int_{A_{J_{k}}}|K(y)|\left|b_{j}(x+y)\right| d y d x \leq c \cdot \sum_{j}\left|B_{j}\right| .
$$

This will follow if we prove that, for every $j$ and $k>\beta_{j}$,

$$
\int_{F_{j, k}} \int_{A_{J_{k}}}|K(y)|\left|b_{j}(x+y)\right| d y d x \leq c \cdot \frac{2^{\beta_{j}}}{2^{k}} \cdot\left|B_{j}\right| .
$$

To prove this, note that if $B_{j} \cap\left(x+\partial A_{J_{k}}\right) \neq \varnothing$, then either $x \in B_{j}+\left\{y|| y \mid=2^{k+1}\right\}$ or $x \in B_{j}+\left\{y|| y \mid=2^{k}\right\}$. The measure of either of these sets is less than a constant multiple of $2^{(d-1) k} \cdot 2^{\beta_{j}}$. Hence we have

$$
\left|F_{j, k}\right| \leq c \cdot 2^{(d-1) k} \cdot 2^{\beta_{j}} .
$$

By the assumption in (1.7), we have the pointwise estimate

$$
\begin{aligned}
\int_{A_{J_{k}}}|K(y)|\left|b_{j}(x+y) d y\right| & =\int_{2^{k} \leq|y|<2^{k+1}}|K(y)|\left|b_{j}(x+y)\right| d y \\
& \leq c \cdot \int_{2^{k} \leq|y|<2^{k+1}} 2^{-k d}\left|b_{j}(x+y)\right| \\
& \leq c \cdot 2^{-k d} \cdot \int\left|b_{j}\right| \\
& \leq c \cdot 2^{-k d} \cdot\left|B_{j}\right|,
\end{aligned}
$$

where in the last step we used property $2(\mathrm{c})$. It follows that

$$
\begin{aligned}
\int_{F_{j, k}} \int_{A_{J_{k}}}|K(y)|\left|b_{j}(x+y)\right| d y d x & \leq\left|F_{j, k}\right| \cdot \sup _{x \in F_{j, k}} \int_{A_{J_{k}}}|K(y)|\left|b_{j}(x+y)\right| d y \\
& \leq c \cdot 2^{(d-1) k} \cdot 2^{\beta_{j}} \cdot 2^{-k d} \cdot\left|B_{j}\right| \\
& =c \cdot \frac{2^{\beta_{j}}}{2^{k}} \cdot\left|B_{j}\right|,
\end{aligned}
$$

finishing the proof of the long inequality, (4.5). 
Let us now prove the short inequality, (4.4). Since the $\ell^{\varrho}$ norm is not greater than the $\ell^{2}$ norm, it is enough to prove that

$$
\left|\left\{\left(\sum_{i \in \mathcal{S}}\left|\sum_{j}\left[B_{j} \cap\left(\partial A_{I_{i}}+x\right) \neq \varnothing\right] K_{I_{i}} b_{j}(x)\right|^{2}\right)^{1 / 2}>1 / 8\right\}\right| \leq c \cdot \sum_{j}\left|B_{j}\right| .
$$

By Markov's inequality, it is enough to prove that

$$
\int_{\tilde{B}^{c}} \sum_{i \in \mathcal{S}}\left|\sum_{j}\left[B_{j} \cap\left(\partial A_{I_{i}}+x\right) \neq \varnothing\right] K_{I_{i}} b_{j}(x)\right|^{2} d x \leq c \cdot \sum_{j}\left|B_{j}\right| .
$$

We want to rewrite this inequality so that we can use the almost orthogonality lemma.

For each $k \in \mathbb{Z}$, collect the indices $i$ for which $I_{i} \subset\left[2^{k}, 2^{k+1}\right)$, and for each $n \in \mathbb{Z}$, collect all $B_{j}$ 's with side length $2^{n-1}$. We can then write

$$
\begin{aligned}
& \sum_{i \in \mathcal{S}}\left|\sum_{j}\left[B_{j} \cap\left(\partial A_{I_{i}}+x\right) \neq \varnothing\right] K_{I_{i}} b_{j}(x)\right|^{2} \\
& =\sum_{k \in \mathbb{Z}} \sum_{i}\left[I_{i} \subset\left[2^{k}, 2^{k+1}\right)\right]\left|K_{I_{i}} \sum_{n} \sum_{j}\left[B_{j} \cap\left(\partial A_{I_{i}}+x\right) \neq \varnothing\right]\left[B_{j} \in \mathbb{L}_{n}\right] b_{j}(x)\right|^{2} .
\end{aligned}
$$

Define $h_{k, n}(x)$ by

$$
h_{k, n}(x)^{2}=\sum_{i}\left[I_{i} \subset\left[2^{k}, 2^{k+1}\right)\right]\left|K_{I_{i}} \sum_{j}\left[B_{j} \cap\left(\partial A_{I_{i}}+x\right) \neq \varnothing\right]\left[B_{j} \in \mathbb{L}_{n}\right] b_{j}(x)\right|^{2} .
$$

Using the triangle inequality and the linearity of the operator $K_{I_{i}}$, we have

$$
\begin{array}{r}
\left(\sum_{i}\left[I_{i} \subset\left[2^{k}, 2^{k+1}\right)\right]\left|K_{I_{i}} \sum_{n} \sum_{j}\left[B_{j} \cap\left(\partial A_{I_{i}}+x\right) \neq \varnothing\right]\left[B_{j} \in \mathbb{L}_{n}\right] b_{j}(x)\right|^{2}\right)^{1 / 2} \\
\leq \sum_{n} h_{k, n}(x) .
\end{array}
$$

So it is sufficient to prove the inequality

$$
\sum_{k} \int_{\tilde{B}^{c}}\left(\sum_{n} h_{k, n}(x)\right)^{2} d x \leq c \cdot \sum_{j}\left|B_{j}\right| .
$$

We just need to make one more definition, and we can recognize the setup for the almost orthogonality lemma. Define

$$
d_{n}=\sum_{B_{j} \in \mathbb{L}_{n}} \mathbf{1}_{B_{j}}
$$

where $\mathbf{1}_{S}=\mathbf{1}_{S}(x)$ is the indicator function of the set $S$.

Since

$$
\sum_{n}\left\|d_{n}\right\|_{L^{2}}^{2}=\sum_{n}\left\|d_{n}\right\|_{L^{1}}=\sum_{j}\left|B_{j}\right|
$$

the inequality to prove can be written as

$$
\sum_{k}\left\|\sum_{n} h_{k, n}(x) \cdot \mathbf{1}_{\tilde{B}^{c}}(x)\right\|_{L^{2}\left(\mathbb{R}^{d}, d x\right)}^{2} \leq c \cdot \sum_{n}\left\|d_{n}\right\|_{L^{2}}^{2} .
$$


By the almost orthogonality lemma, it is sufficient to show that, for every $k, n$,

$$
\int_{\tilde{B}^{c}} h_{k, n}^{2}(x) d x \leq c \cdot 2^{-|k-n|} \cdot\left\|d_{n}\right\|_{L^{2}}^{2} .
$$

Since $x \in \tilde{B}^{c}$ and $h_{k, n}(x)=0$ if $n \geq k$, we need to prove the inequality

$$
\int_{\tilde{B}^{c}} h_{k, n}^{2}(x) d x \leq c \cdot 2^{n-k} \cdot\left\|d_{n}\right\|_{L^{2}}^{2}, \quad n<k .
$$

We will show this by proving, for $x \in \tilde{B}^{c}$, the inequality

$$
h_{k, n}(x)^{2} \leq c \cdot 2^{n-k} \cdot \frac{1}{2^{k d}} \int_{\left.A_{\left[2^{k-1}, 2^{k+2}\right.}\right)} d_{n}(x+y) d y .
$$

Integrating both sides, and using that $d_{n}=d_{n}^{2}$, we obtain the desired inequality.

To make the structure of $h_{k, n}$ more transparent, introduce the sets

$$
P_{i}=\bigcup_{j}\left[B_{j} \in \mathbb{L}_{n}\right]\left\{y \mid y \in B_{j} \cap\left(x+\partial A_{I_{i}}\right), B_{j} \cap\left(x+\partial A_{I_{i}}\right) \neq \varnothing\right\}
$$

and the function $g_{n}=\sum_{j}\left[B_{j} \in \mathbb{L}_{n}\right] b_{j}$. Denoting $\mathcal{S}_{k}=\left\{i \mid i \in \mathcal{S}, I_{i} \in\left[2^{k}, 2^{k+1}\right)\right\}$, we can then write

$$
\begin{aligned}
h_{k, n}(x)^{2} & \leq \sum_{i \in \mathcal{S}_{k}}\left(\int_{P_{i}}|K(y-x)|\left|g_{n}(y)\right| d y\right)^{2} \\
& =\sum_{i \in \mathcal{S}_{k}} \int_{P_{i}}|K(y-x)|\left|g_{n}(y)\right| d y \cdot \int_{P_{i}}\left|K(y-x) \| g_{n}(y)\right| d y .
\end{aligned}
$$

We explicitly wrote out the factor $\int_{P_{i}}|K(y-x)|\left|g_{n}(y)\right| d y$ twice, because we intend to estimate it in two ways.

On the one hand, note that if $y \in P_{i}$, then $y-x \in A_{I_{i}}$, and hence, by assumption (1.7), we have $|K(y-x)| \leq c \cdot 2^{-k d}$. Also, by $2(\mathrm{c})$,

$$
\begin{aligned}
\int_{P_{i}}\left|g_{n}(x+y)\right| d y & \leq \sum_{j}\left[B_{j} \cap\left(\partial A_{I_{i}}+x\right) \neq \varnothing\right] \int_{B_{j}}\left|g_{n}(y)\right| d y \\
& \leq c \cdot \sum_{j}\left[B_{j} \cap\left(\partial A_{I_{i}}+x\right) \neq \varnothing\right]\left[B_{j} \in \mathbb{L}_{n}\right]\left|B_{j}\right| .
\end{aligned}
$$

But the total measure of those $B_{j}$ 's that intersect the boundary of $A_{I_{i}}+x$ and are of side length $2^{n-1}$ is a constant multiple of $2^{(d-1) k} \cdot 2^{n-1}$. Hence we have our first estimate:

$$
\int_{P_{i}}|K(y-x)|\left|g_{n}(y)\right| d y \leq c \cdot 2^{n-k}
$$

We then have, using again that $|K(y-x)| \leq 2^{-k d}$,

$$
h_{k, n}(x)^{2} \leq c \cdot 2^{n-k} \cdot 2^{-k d} \cdot \sum_{i \in \mathcal{S}_{k}} \int_{P_{i}}\left|g_{n}(x+y)\right| d y .
$$


Now just note that, since the $I_{i}, i \in \mathcal{S}_{k}$, form a partition of $\left[2^{k}, 2^{k+1}\right)$,

$$
\begin{aligned}
\sum_{i \in \mathcal{S}_{k}} \int_{P_{i}}\left|g_{n}(x+y)\right| d y & \leq \sum_{j}\left[B_{j} \cap\left(A_{\left[2^{k}, 2^{k+1}\right)}+x\right) \neq \varnothing\right] \int_{B_{j}}\left|d_{n}(y)\right| d y \\
& \leq \int_{A_{\left[2^{k-1,2 k+2}\right)}+x}\left|d_{n}(y)\right| d y
\end{aligned}
$$

as desired. This ends the proof of Theorem B.

Now we are in a position to prove Corollary 1.4 .

Proof of Corollary 1.4 Fix $\varrho>2$ and $1<p<\infty$. We will define a sequence of operators $\left(\mathcal{W}_{\varrho}\right)_{i}=\mathcal{W}_{i}$ which will converge to the variation operator (on $\left.L^{p}\right)$. Note that each operator $\mathcal{W}_{i}$ will be determined if we specify a finite collection of subintervals $\mathcal{I}_{i}=\left\{I_{1}, I_{2}, \ldots, I_{n_{i}}\right\}$. To this end, set

$$
\mathcal{I}_{i}=\left\{\left(k / 2^{i}, j / 2^{i}\right]: 1 \leq k<j \leq 2^{2 i}, k, j \in \mathbb{N}\right\} .
$$

Note that since these partitions refine each other, the operators $\left\{\mathcal{W}_{i}\right\}_{i \geq 1}$, applied to a given $f \in L^{p}$, are (pointwise) increasing.

Now fix $f \in L^{p}\left(\mathbb{R}^{d}\right)$ and let $x \in \mathbb{R}^{d}$. Let $\alpha<\mathcal{V}_{\varrho} f(x)$. There exists a finite partition $\mathcal{J}=\left\{J_{1}, \ldots, J_{n}\right\}$ of $(0, \infty)$ into (nonempty) intervals $J_{j}=\left[a_{j}, b_{j}\right)$ so that

$$
\left(\sum_{j}\left|K_{J_{j}} f(x)\right|^{\varrho}\right)^{1 / \varrho}>\alpha .
$$

By the definition of the $\mathcal{I}_{i}$, we see that for large $i$, each interval in $\mathcal{J}$ will be well-approximated by an interval from $\mathcal{I}_{i}$. That is, for sufficiently large $i$, given any interval in $\mathcal{J}$, there exists some interval in $\mathcal{I}_{i}$ for which the measure of the symmetric difference of this pair of intervals is small. We observe that since $f \in L^{p}$, if $I$ and $J$ are bounded subintervals of $(0, \infty)$ whose symmetric difference has small measure, then $K_{I} f(x)$ and $K_{J} f(x)$ are close. Thus, we may infer the existence of an $i$ so that

$$
\mathcal{W}_{i} f(x)>\alpha \text {. }
$$

Since $\alpha$ is an arbitrary number less than $\mathcal{V}_{\varrho} f(x)$, the sequence $\left(\mathcal{W}_{i} f(x)\right)$ increases to $\mathcal{V}_{\varrho} f(x)$.

We may approach the oscillation operator by a suitable modification of this method. To start, there is a fixed countable partition which determines the boundary intervals for the oscillation, say, $\left\{I_{1}, I_{2}, \ldots\right\}$. Define a sequence of operators $\left(\mathcal{W}_{\varrho}\right)_{n}=\mathcal{W}_{n}$ which will converge to the oscillation by selecting $\left\{I_{1}, I_{2}, \ldots, I_{n}\right\}$ from this sequence for inclusion in $\mathcal{I}_{n}$. If $I_{j}=\left[a_{j}, b_{j}\right)$, add the subintervals of the form $\left[a_{j}, a_{j}+k\left|I_{j}\right| / 2^{n}\right)$ for $k=1,2, \ldots, 2^{n}$ to $\mathcal{I}_{n}$. The arguments go through as before to show that for $f$ in any $L^{p}$ space, the operators $\mathcal{W}_{n} f(x)$ increase pointwise to the oscillation of $f$ at $x$.

Now consider the variation, say, and fix the sequence $\mathcal{W}_{i}$ constructed above. If $K$ satisfies all the hypotheses of Corollary 1.4 then by Theorem A the variation is bounded on $L^{2}$. Thus, by the Monotone Convergence Theorem, the $\mathcal{W}_{i} f(x)$ converge to $\mathcal{V}_{\varrho} f(x)$ in $L^{2}$ as well. In particular, the constants in the type $(2,2)$ bound of the hypothesis of Theorem $\mathrm{B}$ are a priori independent of the collection $\mathcal{I}$, and hence so are the constants in the corresponding weak-type $(1,1)$ bound. The corollary follows. 
The same approach, with the sequence $\mathcal{W}_{i}$ modified as described above, gives the desired conclusion for the oscillation as well.

\section{Derivation of $\lambda$-Jump estimate; ClOSing CONJeCtures}

Proof of Theorem $\left[\right.$ It is clear that $\lambda \Lambda\left(K_{\epsilon}, f, \lambda, x\right)^{\frac{1}{\varrho}} \leq V_{\varrho} f(x)$. Consequently, we have

$$
\left\|\lambda \Lambda\left(K_{\epsilon}, f, \lambda, \cdot\right)^{\frac{1}{\varrho}}\right\|_{p} \leq\left\|V_{\varrho} f\right\|_{p} \leq c(p, \varrho)\|f\|_{p} .
$$

Thus $\left\|\Lambda\left(K_{\epsilon}, f, \lambda, \cdot\right)^{\frac{1}{\varrho}}\right\|_{p} \leq \frac{c(p, \varrho)}{\lambda}\|f\|_{p}$. For the weak-type $(1,1)$ result, we note that

$$
\begin{aligned}
m\left\{x: \Lambda\left(K_{\epsilon}, f, \lambda, x\right)>n\right\} & \leq m\left\{x: \lambda \Lambda\left(K_{\epsilon}, f, \lambda, x\right)^{\frac{1}{\varrho}}>\lambda n^{\frac{1}{\varrho}}\right\} \\
& \leq m\left\{x: V_{\varrho} f(x)>\lambda n^{\frac{1}{\varrho}}\right\} \\
& \leq \frac{c(\varrho)}{\lambda n^{\frac{1}{\varrho}}}\|f\|_{1} .
\end{aligned}
$$

Fix $\alpha<\beta$. Define the number of up-crossings, $N\left(K_{\epsilon}, f, \alpha, \beta, x\right)$, by

$$
\begin{aligned}
& N\left(K_{\epsilon}, f, \alpha, \beta, x\right)=\max \left\{n: \text { there exist } s_{1}<t_{1}<s_{2}<t_{2}<\cdots<s_{n}<t_{n}\right. \\
& \left.\quad \text { such that } K_{s_{i}} f(x)<\alpha, K_{t_{i}} f(x)>\beta, i=1,2, \ldots, n\right\} .
\end{aligned}
$$

Theorem $[$ has the following immediate corollary regarding up-crossings.

Corollary 5.1. Let $\alpha<\beta$. If $\varrho>2$, then

$$
\left\|N\left(K_{\epsilon}, f, \alpha, \beta, \cdot\right)^{\frac{1}{\varrho}}\right\|_{p} \leq \frac{c(\varrho)}{\beta-\alpha}\|f\|_{p}
$$

for $1<p<\infty$, and

$$
m\left\{x: N\left(K_{\epsilon}, f, \alpha, \beta, x\right)>n\right\} \leq \frac{c(\varrho)}{(\beta-\alpha) n^{\frac{1}{\varrho}}}\|f\|_{1} .
$$

Theorem $\mathrm{C}$ and Corollary 5.1 suggest the following:

Conjecture 5.2. The estimates in Theorem $\square$ can be improved to

$$
\left\|\Lambda\left(K_{\epsilon}, f, \lambda, \cdot\right)^{\frac{1}{2}}\right\|_{p} \leq \frac{c(p)}{\lambda}\|f\|_{p}
$$

and

$$
m\left\{x: \Lambda\left(K_{\epsilon}, f, \lambda, x\right)>n\right\} \leq \frac{c}{\lambda n^{\frac{1}{2}}}\|f\|_{1} .
$$

Conjecture 5.3. The estimates in Theorem 5.1 can be improved to

$$
\left\|N\left(K_{\epsilon}, f, \alpha, \beta, \cdot\right)\right\|_{p} \leq \frac{c(p)}{(\beta-\alpha) n}\|f\|_{p}
$$

and

$$
m\left\{x: N\left(K_{\epsilon}, f, \alpha, \beta, x\right)>n\right\} \leq \frac{c}{(\beta-\alpha) n}\|f\|_{1} .
$$

In the case of analogous up-crossing and $\lambda$-jump operators for martingales, differentiation averages, and ergodic averages, we know the improvements conjectured above are possible. However, our current techniques do not allow us to prove these conjectures for singular integral operators. 


\section{Appendix: AuXiliary Results From one Dimension}

In this section we state the main theorems and lemmas from [4], which we apply to obtain the type $(p, p)$ estimates in this paper. The theorems on oscillation and variation for the Hilbert transform are essential in obtaining the estimates for odd kernels, while the lemmas regarding oscillation and variation for certain convolution operators are used in obtaining the estimates for even kernels.

The proofs of all the results stated in this section may be found in 4 .

If $I$ is any subinterval of $(0, \infty)$, set

$$
H_{I} f(x)=\int_{|s| \in I} \frac{f(x-s)}{s} d s,
$$

so that $H_{I} f(x)$ is the truncated Hilbert transform of $f(x)$. The following two theorems are the main results from [4].

Theorem 6.1. The oscillation operator $f \rightarrow O(H f)(x)$, acting on $L^{p}(\mathbb{R})$, is type $(p, p)$ for $1<p<\infty$, and weak-type $(1,1)$.

Theorem 6.2. If $\varrho>2$, then the variation operator $f \rightarrow \mathcal{V}_{\varrho}(H f)$, acting on $L^{p}(\mathbb{R})$, is type $(p, p)$ for $1<p<\infty$, and weak-type $(1,1)$.

The following lemmas also refer to operators acting on functions of a single real variable.

Lemma 6.3. Let $\varphi$ be a differentiable function with support on $[1, \infty)$ such that

(1) $\lim _{s \rightarrow \infty} \varphi(s)=0$, and

(2) $\int_{1}^{\infty} s\left|\varphi^{\prime}(s)\right| d s<\infty$.

Let $\varphi_{t}(x)=\frac{1}{t} \varphi\left(\frac{x}{t}\right)$ and $\varphi_{t} f(x)=\varphi_{t} * f(x)$. Then $f \rightarrow O(\varphi f(x))$ and $f \rightarrow$ $V_{\varrho}(\varphi f(x))(\varrho>2)$ are type $(p, p)$ operators for $1<p<\infty$, and weak-type $(1,1)$ operators.

Lemma 6.4. Let $\varphi$ be a differentiable function with support on $[0,1]$ such that $\int_{0}^{\infty} s\left|\varphi^{\prime}(s)\right| d s<\infty$. Let $\varphi_{t}(x)=\frac{1}{t} \varphi\left(\frac{x}{t}\right)$ and $\varphi_{t} f(x)=\varphi_{t} * f(x)$. Then $f \rightarrow$ $O(\varphi f(x))$ and $f \rightarrow V_{\varrho}(\varphi f(x))(\varrho>2)$ are type $(p, p)$ operators for $1<p<\infty$, and weak-type $(1,1)$ operators.

\section{REFERENCES}

1. M. Akcoglu, R. L. Jones and P. Schwartz, Variation in probability, ergodic theory and analysis, Illinois J. Math. 42 (1998) 154-177. MR 99a:60048

2. A. P. Calderón and A. Zygmund, On the existence of certain singular integrals, Acta. Math. (1952) 88, 85-139. MR 14:637f

3. A. Calderón and A. Zygmund, On singular integrals Amer. J. Math, 78 (1956), 289-309. MR 18:894a

4. J. Campbell, R. Jones, K. Reinhold, and M. Wierdl, Oscillation and Variation for the Hilbert Transform, Duke Math. J. 105 (2000), 59-83. MR 2001h:14021

5. M. Cotlar, A unified theory of Hilbert transforms and ergodic theorems, Rev. Math. Cuyana, 1 (1955), 105 - 167. MR 18:893d

6. M. Christ and J. L. Rubio de Francia Weak-type $(1,1)$ bounds for rough operators II, Invent. Math. 93 (1988), no. 1, 225-237. MR 90d:42021

7. R. Fefferman, A theory of entropy in Fourier analysis, Adv. in Math., 30(1978) 171-201. MR 81g: 42022

8. R. Jones, I. Ostrovskii, and J. Rosenblatt, Square functions in ergodic theory, Ergodic Theory and Dyn. Sys. 16 (1996), 267-305. MR 97f:28044 
9. R. Jones, R. Kaufmann, J. Rosenblatt and M. Wierdl, Oscillation in ergodic theory, Ergodic Theory and Dyn. Sys. 18 (1998), 889-935. MR 2000b:28019

10. R. Jones, J. Rosenblatt, and M. Wierdl, Oscillation in ergodic theory: higher dimensional results, preprint, to appear in Israel J. of Math.

11. A. W. Knapp and E. M. Stein, Intertwining operators on semi-simple groups, Ann. of Math. 93 (1971), 489 - 578. MR 57:536

12. K. Petersen, Another proof of the existence of the ergodic Hilbert transform, Proc. Amer. Math. Soc. 88, 39-44. MR 84i:28022

13. J. Qian, The p-variation of partial sum processes and the empirical process, Ann. of Prob., (1998), 26, 1370-1383. MR 99i:60052

14. M. Riesz, Sur les fonctions conjugées, Math. Zeit. 27 (1927), 218-244.

15. E. M. Stein, Singular integrals and differentiability properties of functions, Princeton University Press, Princeton, N. J., 1970. MR 44:7280

16. E. M. Stein and S. Wainger, Discrete analogues of singular Radon transforms, Bull. Amer. Math. Soc. (N.S.) 23 (1990), no. 2, 537-544. MR 92e:42010

17. E. M. Stein and G. Weiss, Introduction to Fourier Analysis on Euclidean spaces, Princeton University Press, Princeton, N. J., 1971. MR 46:4102

18. S. Wainger, Discrete analogues of singular and maximal Radon transforms, Doc. Math., Extra Vol. ICM (1998), 743-753. MR 99g:44005

Department of Mathematical Sciences, Dunn Hall 373, University of Memphis, MemPhis, TENNESSEE 38152

E-mail address: jtc@campbeljpc2.msci.memphis.edu

Department of Mathematics, DePaul University, 2219 N. Kenmore, Chicago Illinois 60614

E-mail address: rjones@condor.depaul.edu

Department of Mathematics, University at Albany, SUny, 1400 Washington Ave., Albany, NeW York 12222

E-mail address: reinhold@csc.albany.edu

Department of Mathematical Sciences, Dunn Hall 373, University of Memphis, MemPHIS, TENNESSEE 38152

E-mail address: mw@moni.msci.memphis.edu 